PROCEEDINGS OF THE

AMERICAN MATHEMATICAL SOCIETY

Volume 125, Number 8, August 1997, Pages 2207-2216

S 0002-9939(97)03799-4

\title{
INFINITE DIMENSIONAL QUADRATIC FORMS ADMITTING COMPOSITION
}

\author{
ALBERTO ELDUQUE AND JOSÉ MARÍA PÉREZ
}

(Communicated by Lance W. Small)

\begin{abstract}
Composition algebras with a unit element constitute a well-known class of algebras. In this paper, those composition algebras with a one-sided unit element are characterized and examples are given of arbitrary infinite dimension.
\end{abstract}

\section{INTRODUCTION}

This paper bears the same title as $[\mathrm{K}]$ and the results here may be considered as an extension of the results there. Let $F$ be an arbitrary ground field, $A$ a vector space over $F, q: A \rightarrow F$ a quadratic form, so that $q(\alpha x)=\alpha^{2} q(x)$ for any $\alpha \in F$ and $x \in A$, and the function $A \times A \rightarrow F$ given by $q(x, y)=q(x+y)-q(x)-q(y)$ is a (symmetric) bilinear form. We say that the quadratic form $q$ is nondegenerate if $\{x \in A: q(x)=q(x, A)=0\}=0$ and we say that it is strictly nondegenerate (see [ZSSS]) if $\{x \in A: q(x, A)=0\}=0$. If the ground field is of characteristic $\neq 2$ then $q(x)=\frac{1}{2} q(x, x)$, so both definitions coincide. A nondegenerate quadratic form is said to admit composition in case there is a bilinear product $x y$ from $A \times A$ to $A$ with

$$
q(x y)=q(x) q(y)
$$

for any $x, y \in A$. In this case, $A$ is said to be a composition algebra relative to $q$.

The result proved in $[\mathrm{K}]$ extends Hurwitz's classical theorem ([H1]) and asserts that if $A$ is an algebra with unit element $e$ over a field $F$ and carries a nondegenerate quadratic form $q$ satisfying (1), then either the characteristic of $F$ is $2, q(A, A)=0$ and $A$ is a purely inseparable field extension of $F$ with $x^{2}=q(x) e$ for any $x \in A$, or $q$ is strictly nondegenerate and $A$ is a finite dimensional classical composition algebra of dimension 1, 2, 4 or 8. These latter algebras are described in [ZSSS, Chapter 2].

Kaplansky's result was preceded by an analogous result of Wright ([W]) asserting that any real absolute valued division algebra with a unit element is finite dimensional and thus it is either the real or complex field, or the division algebras of quaternions or octonions. Wright's result extended a previous result by Albert [A] where the extra hypothesis of the algebra being algebraic was imposed. Recall that

Received by the editors September 29, 1995 and, in revised form, February 6, 1996.

1991 Mathematics Subject Classification. Primary 17A75, $11 \mathrm{E} 88$.

The second author was supported by a grant from the 'Plan de Formación del Personal Investigador', DGICYT.

Work supported by the Spanish DGICYT (PB 94-1311-C03-03). 
an absolute valued real algebra is an algebra over the real field equipped with a norm $|$.$| such that |x y|=|x||y|$ for any $x, y$. Later on, Urbanik and Wright [U-W] were able to remove the assumption that the absolute valued algebra is a division algebra, and also provided examples of infinite dimensional real absolute valued algebras showing that the presence of unit element is essential.

Actually, in $[\mathrm{K}]$ it is shown that even if the composition algebra $A$ has no unit element but there is an element $a \in A$ with $q(a)=1$ and such that the left and right multiplications by $a$, denoted respectively by $L_{a}$ and $R_{a}$, are bijections, then the quadratic form $q$ also admits composition with the new product

$$
x \cdot y=R_{a}^{-1}(x) L_{a}^{-1}(y),
$$

and the element $e=a^{2}$ is the unit element for this new product, so the previous result applies. This always happens in the finite dimensional case or if $A$ is a division algebra.

This can be extended a little further:

Proposition 1. Let $A$ be a composition algebra over the field $F$ relative to the nondegenerate quadratic form $q$ and assume that there are elements $a$ and $b$ in $A$ such that $L_{a}$ and $R_{b}$ are bijective. Then either the characteristic of $F$ is 2, $q(A, A)=0$ and $A$ is a purely inseparable field extension of $F$ of exponent 1 with $x^{2}=q(x) 1$ for any $x \in A$, or $q$ is strictly nondegenerate and $A$ is finite dimensional, the dimension being restricted to 1, 2, 4 or 8 .

Proof. First notice that because of (1) $q(a) \neq 0 \neq q(b)$, so also $q(a b) \neq 0$. We define a new product in $A$ by means of

$$
x \cdot y=R_{b}^{-1}(x) L_{a}^{-1}(y)
$$

and a new quadratic form $\tilde{q}(x)=\frac{1}{q(e)} q(x)$ with $e=a b$. Then notice that $e$ is the unit element of the algebra $(A, \cdot)$ and that

$$
\tilde{q}(x \cdot y)=\frac{1}{q(e)} q\left(R_{b}^{-1}(x)\right) q\left(L_{a}^{-1}(y)\right)=\frac{1}{q(e)} \frac{q(x)}{q(b)} \frac{q(y)}{q(a)}=\tilde{q}(x) \tilde{q}(y),
$$

so $(A, \cdot)$ is a composition algebra with unit element relative to $\tilde{q}$. By Kaplansky's result mentioned earlier either $\tilde{q}$ is strictly nondegenerate (and so is $q$ ), and the dimension of $A$ is finite and restricted to $1,2,4$ or 8 , or the characteristic of $F$ is 2, $q(A, A)=\tilde{q}(A, A)=0$ and $(A, \cdot)$ is a purely inseparable field extension of $F$ with $x \cdot x=\tilde{q}(x) e$ for any $x \in A$. In the latter case, $q(x+y)=q(x)+q(y)$ for any $x, y$ and since $q$ is nondegenerate, $q: A \rightarrow F$ is a one-to-one ring homomorphism. In particular, $A$ is commutative and associative, so $x \cdot y=L_{a}^{-1} R_{b}^{-1}(x y)=L_{e}^{-1}(x y)$. Take $u=L_{e}^{-1}(e)$; then $u x=L_{e}^{-1}(e) x=e \cdot x=x$, so $u$ is the unit element of $A$, which implies $q(u)=1$. Moreover, for any $x \in A, x^{2}=e\left(L_{e}^{-1}\left(x^{2}\right)\right)=e(x \cdot x)=$ $e(\tilde{q}(x) e)=q(x)\left(\frac{1}{q(e)} e^{2}\right)$. In particular, $u=u^{2}=\frac{1}{q(e)} e^{2}$, so that $x^{2}=q(x) u$. Therefore for any $0 \neq x \in A$, we have $q(x) \neq 0$ since $q$ is nondegenerate and it follows that $x$ has an inverse and $A$ is a field.

For real absolute valued algebras, the existence of elements $a$ and $b$ with $L_{a}$ and $R_{b}$ bijections was shown in $[\mathrm{RP}]$ to imply the finite dimensionality of the algebra, but with a completely different method. Notice that the argument used in the proof above to pass to a unital composition algebra is also valid for absolute valued algebras, thus providing a simplification of [RP, Proposition 3]. Notice also that in 
a finite dimensional composition algebra $L_{a}$ is bijective if and only if so is $R_{a}$ (if and only if $q(a) \neq 0)$.

This paper is devoted to studying what happens if we assume only the existence of an element $a$ in a composition algebra $A$ with bijective $L_{a}$. It will be shown that $A$ may have any infinite dimension and examples will be constructed over arbitrary fields.

The next result ([RP, Proposition 4, Theorems 2 and 3$])$ is a motivation for our results:

Theorem 2 (Rodríguez-Palacios). Let $A$ be an absolute valued real algebra. Then:

i) If there is an element $a \in A$ such that $L_{a}$ is invertible, then $L_{x}$ is invertible for any nonzero $x \in A$. In this case, $A$ is isotopic (in a specific sense) to an absolute valued real algebra with a left unit $e$ (that is, $L_{e}=1$ ).

ii) If $A$ contains a left unit $e$ then the norm in $A$ is the norm associated to an inner product, so $A$ is a real composition algebra with positive definite quadratic form q. Besides, it satisfies:

$$
q(x y, z)+q(y, x z)=0 \quad \text { and } \quad x(x y)=-q(x) y
$$

for any $x$ orthogonal to $e$ and any $y \in A$.

iii) There exist Hilbert spaces of arbitrary infinite Hilbertian dimension which become absolute valued algebras with left unit under a suitable multiplication.

The proof of iii) in the theorem above requires the use of the so-called Fermi-Fock space associated to a nonzero complex Hilbert space and the "canonical anticommutation relations" for quantum mechanics. A previous construction of an absolute valued algebra with left unit over the real infinite dimensional separable Hilbert space has been given in $[\mathrm{C}]$.

There are examples showing that the results valid for absolute valued algebras do not always have counterparts for composition algebras over arbitrary fields. For instance, any flexible absolute valued algebra is finite dimensional [EIM-M], but there are infinite dimensional commutative (hence flexible) composition algebras over arbitrary fields (of characteristic $\neq 2$ ) $[\mathrm{E}-\mathrm{M}]$. However, with completely different techniques, although very much inspired by the work of Rodríguez Palacios $[\mathrm{RP}]$, we will prove analogous results to the ones in Theorem 2 for composition algebras over arbitrary fields. Our construction will provide also examples of real absolute valued algebras with left unit and with arbitrary infinite dimension (not Hilbertian dimension).

Our approach will follow the treatment in [L] of the problem solved by Hurwitz, in his posthumous 1923 paper [H2], and independently by Radon in [R]: "to find all pairs of positive integers $(n, p)$ and bilinear multiplications $\mathbb{R}^{p} \times \mathbb{R}^{n} \rightarrow \mathbb{R}^{n}$, $\left(\left(x_{1}, \ldots, x_{p}\right),\left(y_{1}, \ldots, y_{n}\right)\right) \mapsto\left(z_{1}, \ldots, z_{n}\right)$, such that

$$
\left(x_{1}^{2}+\cdots+x_{p}^{2}\right)\left(y_{1}^{2}+\cdots y_{n}^{2}\right)=z_{1}^{2}+\cdots+z_{n}^{2}
$$

for any $x_{1}, \ldots, x_{p}, y_{1}, \ldots, y_{n} \in \mathbb{R}^{\prime \prime}$ (see $[\mathrm{L}, \mathrm{S} 1, \mathrm{~S} 2, \mathrm{~S} 3, \mathrm{~S} 4, \mathrm{~W}-\mathrm{S}, \mathrm{E}-\mathrm{R}, \mathrm{M}, \mathrm{F}, \mathrm{Ju}, \mathrm{Z}]$ for work on this subject).

In order to deal with fields of arbitrary characteristic we will make use of the Clifford algebra of a quadratic space with base point as defined in $[\mathrm{J}]$ in connection with the special universal envelope of certain quadratic Jordan algebras, instead of the usual Clifford algebras used in [L]. 


\section{The Composition PRoblem And Clifford Algebras}

Let $(U, q)$ and $(V, Q)$ be vector spaces over an arbitrary ground field $F$, equipped with nondegenerate quadratic forms $q$ and $Q$, and assume that there is a bilinear map

$$
\begin{aligned}
\lambda: U \times V & \longrightarrow V, \\
(a, v) & \mapsto a \cdot v
\end{aligned}
$$

such that

$$
Q(a \cdot v)=q(a) Q(v)
$$

for any $a \in U$ and $v \in V$. By linearizing (4) we obtain

$$
\begin{aligned}
& Q(a \cdot u, a \cdot v)=q(a) Q(u, v), \\
& Q(a \cdot v, b \cdot v)=q(a, b) Q(v), \\
& Q(a \cdot u, b \cdot v)+Q(b \cdot u, a \cdot v)=q(a, b) Q(u, v)
\end{aligned}
$$

for any $a, b \in U$ and $u, v \in V$. Define for any $a \in U$ the linear map $\lambda_{a}: V \rightarrow V$ by $\lambda_{a}(v)=a \cdot v$. We will denote again by $\lambda$ the linear map $\lambda: U \rightarrow \operatorname{End}_{F}(V)$ $\left(a \mapsto \lambda_{a}\right)$. This map is one-to-one since $\lambda_{a}=0$ implies by (4) that $q(a)=0$ and by (6) that $q(a, A)=0$, so $a=0$ since $q$ is nondegenerate. Finally assume that there is an element $e \in U$ such that $\lambda_{e}$ is a bijection. By (4) this implies that $q(e) \neq 0$. Now (5) with $b=e$ and $v^{\prime}=e \cdot v$ gives

$$
Q\left(\lambda_{e}(u), v^{\prime}\right)=Q\left(u, q(e) \lambda_{e}^{-1}\left(v^{\prime}\right)\right)
$$

so that $\lambda_{e}$ has an adjoint map $\lambda_{e}^{*}$ relative to $Q$ and moreover $\lambda_{e}^{*}=q(e) \lambda_{e}^{-1}$. By $(7)$ with $b=e$ and $v^{\prime}=e \cdot v$

$$
Q\left(\lambda_{a}(u), v^{\prime}\right)+Q\left(\lambda_{e}(u), \lambda_{a} \lambda_{e}^{-1}\left(v^{\prime}\right)\right)=q(a, e) Q\left(u, \lambda_{e}^{-1}\left(v^{\prime}\right)\right),
$$

that is,

$$
\begin{aligned}
Q\left(\lambda_{a}(u), v^{\prime}\right) & =Q\left(u,\left(q(a, e) \lambda_{e}^{-1}-\lambda_{e}^{*} \lambda_{a} \lambda_{e}^{-1}\right)\left(v^{\prime}\right)\right) \\
& =Q\left(u, \lambda_{e}^{*}\left(\frac{q(a, e)}{q(e)} \lambda_{e}-\lambda_{a}\right) \lambda_{e}^{-1}\left(v^{\prime}\right)\right) \\
& =Q\left(u, \lambda_{e}^{*} \lambda_{\bar{a}} \lambda_{e}^{-1}\left(v^{\prime}\right)\right)
\end{aligned}
$$

where $\bar{a}=\frac{q(a, e)}{q(e)} e-a$, so $\overline{\bar{a}}=a$ for any $a \in U$. Notice that the map $a \mapsto \bar{a}$ is the reflection through the line $F e$, so that it is an isometry: $q(\bar{a})=q(a)$ for any $a \in U$. Therefore we conclude that for any $a \in U, \lambda_{a}$ has an adjoint $\lambda_{a}^{*}$ relative to $Q$ and that $\lambda_{a}^{*}=\lambda_{e}^{*} \lambda_{\bar{a}} \lambda_{e}^{-1}$ or equivalently

$$
\lambda_{a}^{*} \lambda_{e}=\lambda_{e}^{*} \lambda_{\bar{a}}
$$

for any $a \in U$. Now (5) implies that

$$
Q\left(u,\left(q(a) 1-\lambda_{a}^{*} \lambda_{a}\right)(v)\right)=0,
$$

for any $u, v \in V$, and it can be easily proved using (4), the expression $\lambda_{a}^{*}=$ $\lambda_{e}^{*} \lambda_{\bar{a}} \lambda_{e}^{-1}=q(e) \lambda_{e}^{-1} \lambda_{\bar{a}} \lambda_{e}^{-1}$ and $q(\bar{a})=q(a)$, that $Q\left(\lambda_{a}^{*}(v)\right)=q(a) Q(v)$ for any $v \in V$, and from here that

$$
Q\left(\left(q(a) 1-\lambda_{a}^{*} \lambda_{a}\right)(v)\right)=0
$$


for any $v \in V$. By the nondegeneracy of $Q$, it follows that

$$
\lambda_{a}^{*} \lambda_{a}=q(a) 1,
$$

so that $\lambda_{e}^{*} \lambda_{\bar{a}} \lambda_{e}^{-1} \lambda_{a}=q(a) 1$ or $\lambda_{\bar{a}} \lambda_{e}^{-1} \lambda_{a}=\frac{q(a)}{q(e)} \lambda_{e}$ for any $a \in U$; thus $\lambda_{a} \lambda_{e}^{-1} \lambda_{\bar{a}}=$ $\frac{q(\bar{a})}{q(e)} \lambda_{e}=\frac{q(a)}{q(e)} \lambda_{e}$ and we conclude that $\lambda_{a}$ is invertible if and only if $q(a) \neq 0$ and in this case $\lambda_{a}^{*}=q(a) \lambda_{a}^{-1}$.

We can define a new bilinear map

$$
\begin{aligned}
\tilde{\lambda}: U \times V & \longrightarrow V, \\
(a, v) & \mapsto a \circ v
\end{aligned}
$$

by $a \circ v=\tilde{\lambda}_{a}(v)=\lambda_{a} \lambda_{e}^{-1}(v)$ for any $a \in U$ and $v \in V$. Then $Q(a \circ v)=$ $q(a) Q\left(\lambda_{e}^{-1}(v)\right)=\tilde{q}(a) Q(v)$ where $\tilde{q}: A \rightarrow F$ is the quadratic form given by $\tilde{q}(a)=$ $\frac{q(a)}{q(e)}$. Moreover, $\tilde{\lambda}_{e}=1$, so the arguments above show that for any $a \in U, \tilde{\lambda}_{a}$ has an adjoint $\tilde{\lambda}_{a}^{*}$ relative to $Q$ and $\tilde{\lambda}_{a}^{*}=\tilde{\lambda}_{\bar{a}}$. We summarize this work in the next theorem which should be compared with parts i) and ii) of Theorem 2:

Theorem 3. Let $(U, q)$ and $(V, Q)$ be vector spaces over an arbitrary ground field $F$ equipped with nondegenerate quadratic forms $q$ and $Q$ and assume that there is a bilinear map $\lambda: U \times V \rightarrow V\left((a, v) \mapsto \lambda(a, v)=\lambda_{a}(v)=a \cdot v\right)$ such that $Q(a \cdot v)=q(a) Q(v)$ for any $a \in U$ and $v \in V$. Then:

i) If there is an element $e \in U$ such that $\lambda_{e}$ is a bijection then for any $a \in U, \lambda_{a}$ is a bijection if and only if $q(a) \neq 0$. Moreover, by defining $a \circ v=a \cdot\left(\lambda_{e}^{-1}(v)\right)$ and $\tilde{q}=\frac{1}{q(e)} q$ a new 'multiplication' $\tilde{\lambda}: U \times V \rightarrow V$ is obtained with $Q(a \circ v)=\tilde{q}(a) Q(v)$ and with $\tilde{\lambda}_{e}=1$.

ii) If there is an element $e \in U$ with $\lambda_{e}=1$, then $q(e)=1$ and for any $a \in U$ and $u, v \in V$ :

$$
Q(a \cdot u, v)=Q(u, \bar{a} \cdot v), \quad \bar{a} \cdot(a \cdot u)=a \cdot(\bar{a} \cdot u)=q(a) u,
$$

where $a \mapsto \bar{a}=\frac{q(a, e)}{q(e)} e-a$ is the reflection through the line Fe.

Assume now that $(U, q),(V, Q), \lambda$ and $e \in U$ are as in Theorem 3 with $\lambda_{e}=1$. Notice that for any $a \in U, q(a) 1=\lambda_{a}^{*} \lambda_{a}=\lambda_{\bar{a}} \lambda_{a}=\left(q(a, e) \lambda_{e}-\lambda_{a}\right) \lambda_{a}=q(a, e) \lambda_{a}-$ $\lambda_{a}^{2}$, so

$$
\lambda_{a}^{2}-q(a, e) \lambda_{a}+q(a) 1=0 .
$$

As in $[\mathrm{J}]$, we define the Clifford algebra $C(U, q, e)$ as the quotient of the tensor algebra $T(U)$ modulo the ideal generated by the elements $1-e$ and $a \otimes a-q(a, e) a+$ $q(a) 1$ for $a \in U$. It follows easily from the definitions that if the characteristic is $\neq 2$ and $U^{\prime}$ is the orthogonal subspace to $e$ relative to $q$, then $C(U, q, e)$ is isomorphic to the usual Clifford algebra $C\left(U^{\prime},-q\right)$ (which is the quotient of $T\left(U^{\prime}\right)$ modulo the ideal generated by the elements $a \otimes a+q(a) 1$, with $\left.a \in U^{\prime}\right)$.

In the setting of [J, Chapter 2], $\lambda$ is an associative specialization of the quadratic Jordan algebra associated to $(U, q, e)$ and $C(U, q, e)$ is the special universal envelope of this Jordan algebra. Also, there is a canonical injective map $U \rightarrow C(U, q, e)$ ([J, 2.2.17]) so we will identify $U$ with its image. The reflection $a \mapsto \bar{a}$ is easily seen to extend to an involution of $C(U, q, e)$. From its very definitions $a \bar{a}=\bar{a} a=q(a) 1$ in $C(U, q, e)$ and, by linearization, $\bar{a} b+\bar{b} a=q(a, b) 1$ for any $a, b \in U$. 
Theorem 4. Let $(U, q)$ and $(V, Q)$ be vector spaces over an arbitrary ground field $F$ equipped with nondegenerate quadratic forms $q$ and $Q$. Let $\lambda: U \times V \rightarrow V$ $((a, v) \mapsto a \cdot v)$ be a bilinear map and let $e \in U$ be an element with $q(e)=1$ and $\lambda_{e}=1$. Then:

a) If $Q(a \cdot v)=q(a) Q(v)$ for any $a \in U$ and $v \in V$, then $\lambda$ induces a homomorphism of associative algebras $\lambda: C(U, q, e) \rightarrow \operatorname{End}_{F}(V)$ which verifies for any $a, b$ in a fixed basis of $U$ and any $v$ in a fixed basis of $V$ :

i) $Q\left(\lambda_{a}(v)\right)=q(a) Q(v)$,

ii) $Q\left(\lambda_{a}(v), \lambda_{b}(v)\right)=q(a, b) Q(v)$ and

iii) $\lambda_{a}$ has an adjoint $\lambda_{a}^{*}$ relative to $Q$ and $\lambda_{a}^{*}=\lambda_{\bar{a}}$, where $\bar{a}=q(a, e) e-a$. Moreover, if the characteristic is $\neq 2$, i) and ii) are consequences of iii).

b) Conversely, given a homomorphism $\lambda: C(U, q, e) \rightarrow \operatorname{End}_{F}(V)$ satisfying the extra conditions i), ii) and iii) above, then the bilinear map $U \times V \rightarrow V$ defined by $a \cdot v=\lambda_{a}(v)$ verifies $Q(a \cdot v)=q(a) Q(v)$ for any $a \in U$ and $v \in V$.

Proof. For a) notice that (10) and the definition of $C(U, q, e)$ immediately give the representation $\lambda: C(U, q, e) \rightarrow \operatorname{End}_{F}(V)$, which verifies i), ii) and iii). If the characteristic is $\neq 2, a, b \in U, v \in V$ and iii) is satisfied then $\lambda_{a}$ has an adjoint $\lambda_{a}^{*}$ relative to $Q$ and $\lambda_{a}^{*}=\lambda_{\bar{a}}$ for any $a \in U$ (not just for the elements in a fixed basis). For any $a \in U$ and $v \in V$

$$
\begin{aligned}
Q\left(\lambda_{a}(v)\right) & =\frac{1}{2} Q\left(\lambda_{a}(v), \lambda_{a}(v)\right)=\frac{1}{2} Q\left(\lambda_{\bar{a}} \lambda_{a}(v), v\right) \\
& =\frac{1}{2} Q\left(\left(q(a, e) \lambda_{a}-\lambda_{a}^{2}\right)(v), v\right)=\frac{q(a)}{2} Q(v, v) \\
& =q(a) Q(v),
\end{aligned}
$$

and linearizing this we obtain ii). Hence in this case, i) and ii) are consequences of iii).

For b) notice that for $a, b$ in the fixed basis of $U$ and $u, v$ in the fixed basis of $V, Q(a \cdot u, a \cdot v)=Q\left(\lambda_{\bar{a}} \lambda_{a}(u), v\right)=q(a) Q(u, v)$ and $Q(a \cdot u, b \cdot v)+Q(b \cdot u, a \cdot v)=$ $Q\left(\lambda_{\bar{b} a+\bar{a} b}(u), v\right)=q(a, b) Q(u, v)$. Hence (4) and its linearizations are satisfied for basic elements and therefore $Q(a \cdot v)=q(a) Q(v)$ for any $a \in U$ and $v \in V$.

In case the characteristic is $\neq 2$, a related result for finite dimensional spaces appears in [S1, 3.1].

\section{COMPOSITION ALGEBRAS With INVERTIBLE LEFT MULTIPLICATIONS}

Theorem 5. Let $A$ be a composition algebra relative to the (nondegenerate) quadratic form $q$ and assume there is an element $e \in A$ such that $L_{e}$ is a bijection. Then either i) the characteristic of $F$ is $2, q(A, A)=0$ and $A$ is a purely inseparable field extension of $F$, with $x^{2}=q(x) 1$ for any $x$, or ii) $q$ is strictly nondegenerate and the dimension of $A$ is restricted to be 1,2,4,8 or infinite.

Proof. Assume first that $L_{e}=1$, so $q(e)=1$ and let $S=\{x \in A: q(x, A)=0\}$. Suppose that $S \neq 0$, so that the characteristic of $F$ is 2 , and take $0 \neq x \in S$. By (6), for any $z \in A, 0=q(x, z x)=q(e x, z x)=q(e, z) q(x)$ and since $q(x) \neq 0$ because $q$ is nondegenerate, we conclude that $q(e, A)=0$, that is, $e \in S$. Now by (7) $q(y x, z)=q(y x, e z)=q(e x, y z)+q(y, e) q(x, z)=q(x, y z)=0$ for any $y, z \in A$ and $x \in S$; thus $A S \subseteq S$. Take now $a \in A$ with $q(a)=0$. Then $q(a e)=q(a) q(e)=0$ and $a e \in A S \subseteq S$; hence $a e=0$ since $q$ is nondegenerate. 
But then $0=q(a e, z e)=q(e) q(a, z)=q(a, z)$ for any $z$, so $q(a)=q(a, A)=0$ and $a=0$. Therefore $q(a) \neq 0$ for any $a \neq 0$. But for any $z \in A, q(z e+e z)=$ $q(z e)+q(e z)+q(z e, e z)=2 q(z) q(e)+q(z e, e z)=0$ since $z e \in A S \subseteq S$. Hence $e z+z e=0, z e=e z=z$ and $e$ is the unit element of $A$. Now as in [K, proof of the Theorem], $q: A \rightarrow F$ is a one-to-one ring homomorphism, so $A$ is an integral domain and since $q\left(x^{2}\right)=q(x)^{2}=q(q(x) e)$ we obtain that $x^{2}=q(x) e$ for any $x \in A$ and i) follows in this case.

If we only assume that $L_{e}$ is a bijection, so $q(e) \neq 0$, we consider the new product on $A$ given by $x \cdot y=x\left(L_{e}^{-1} y\right)$ and the new quadratic form $\tilde{q}(x)=\frac{q(x)}{q(e)}=q\left(L_{e}^{-1} x\right)$. Then $\tilde{q}(x \cdot y)=\frac{1}{q(e)} q\left(x\left(L_{e}^{-1} y\right)\right)=\frac{1}{q(e)} q(x) q\left(L_{e}^{-1} y\right)=\tilde{q}(x) \tilde{q}(y)$. Since $S=\{x \in$ $A: q(x, A)=0\}=\{x \in A: \tilde{q}(x, A)=0\}$ and $e \cdot x=x$ for any $x \in A$, if $S \neq 0$ we conclude that $(A, \cdot)$ is a purely inseparable field extension of $F$ and $e$ is the unit element of $(A, \cdot)$. Thus $x=x \cdot e=x u$ with $u=L_{e}^{-1} e$, so $u$ is a right unit of $A$. The argument in the first paragraph of this proof with $L_{e}=1$ substituted by $R_{u}=1$ shows that $A$ is a purely inseparable field extension of $F$ with unit element $u$. Alternatively we could have used Proposition 1 . Therefore we obtain i) in case $q$ is not strictly nondegenerate.

Finally, if $q$ is strictly nondegenerate, $L_{e}$ is a bijection and the dimension of $A$ is finite, then $q(e) \neq 0$, so $R_{e}$ is also a bijection and Proposition 1 implies that the dimension of $A$ is restricted to $1,2,4$ or 8 .

As remarked in $[\mathrm{K}]$, purely inseparable extensions of any infinite dimension or any finite dimension power of 2 exist over suitable ground fields. We finish the paper showing the existence of composition algebras of arbitrary infinite dimension over arbitrary ground fields with strictly nondegenerate quadratic form as in part ii) of Theorem 5. In order to do this and because of Theorem 4, we need to find suitable representations $\lambda: C(U, q, e) \rightarrow \operatorname{End}_{F}(U)$ or, alternatively, $\lambda: C(U, q, e) \rightarrow$ $\operatorname{End}_{F}(V)$ with $(U, q)$ isomorphic to $(V, Q)$ as vector spaces with quadratic forms. The most natural representation of $C(U, q, e)$ is the left regular representation which assigns to each element $a \in C(U, q, e)$ the left multiplication $L_{a}$. We will take this into account.

First a trivial remark: if $V$ is a vector space over a field $F,\left\{v_{i}: i \in I\right\}$ is a basis of $V$ and $V^{*}$ denotes the dual vector space, then $\hat{V}=\left\{\varphi \in V^{*}: \varphi\left(v_{i}\right)=0\right.$ for all but a finite number of indices $i \in I\}$ is a subspace of $V^{*}$. Moreover, define $\varphi_{i} \in V^{*}$ for any $i \in I$ by $\varphi_{i}\left(v_{j}\right)=\delta_{i j}$ as usual. Then $\left\{\varphi_{i}: i \in I\right\}$ is a basis of $\hat{V}$. Notice that the definition of $\hat{V}$ depends in general on the given basis.

Example 1. Let $(U, q, e)$ be defined by $U=(F e \oplus F f) \oplus\left(\oplus_{i \in I}\left(F e_{i} \oplus F f_{i}\right)\right)$ with an infinite well ordered index set $I$ and where the quadratic form $q$ is determined by $q(e)=1, q(f)=0=q\left(e_{i}\right)=q\left(f_{i}\right)$ for any $i \in I$ and $q(e, f)=1=q\left(e_{i}, f_{i}\right)$ for any $i$ and 0 otherwise. Hence $(U, q, e)$ is an orthogonal sum of hyperbolic planes, the number of which is the cardinality of $I$.

Let $W=C(U, q, e)$ which has a basis formed by the elements

$$
f^{\epsilon} e_{i_{1}}^{\alpha_{i_{1}}} f_{i_{1}}^{\beta_{i_{1}}} \cdots e_{i_{r}}^{\alpha_{i_{r}}} f_{i_{r}}^{\beta_{i_{r}}}
$$

with $r \geq 0, \epsilon, \alpha_{i_{j}}$ and $\beta_{i_{j}}$ are either 0 or $1, i_{1}<\cdots<i_{r}$ and $\alpha_{i_{j}}+\beta_{i_{j}} \neq 0$ for any $j=1, \ldots, r$, where $a^{0}=e, a^{1}=a$ for any $a$. Since $I$ is infinite, the cardinality of this basis is the same cardinality of $I$. Consider also the dual space $W^{*}$, its subspace $\hat{W}$ relative to the basis above and the mappings $\mu: U \rightarrow \operatorname{End}_{F}(W)$, 
$a \mapsto \mu_{a}$, where $\mu_{a}$ is the left multiplication by $a$ in the algebra $C(U, q, e)$, and $\mu^{*}: U \rightarrow \operatorname{End}_{F}\left(W^{*}\right), a \mapsto \mu_{a}^{*}$, where $\mu_{a}^{*}(\varphi)(w)=\varphi\left(\mu_{\bar{a}}(w)\right)$, with $\bar{a}=q(a, e) e-a$. By its own definition $e$ is the unit element of $C(U, q, e), f^{2}=f, f e_{i}+e_{i} f=e_{i}$, $f f_{i}+f_{i} f=f_{i}, e_{i} f_{i}+f_{i} e_{i}=-e$ for any $i \in I$, and $e_{i} e_{j}=-e_{j} e_{i}, f_{i} f_{j}=-f_{j} f_{i}$, $e_{i} f_{j}=-f_{j} e_{i}$ for $i \neq j$. From this it follows that $\mu_{a}^{*}(\varphi) \in \hat{W}$ if $\varphi \in \hat{W}$, we leave the details to the reader.

Finally, let $V=W \times \hat{W}$, equipped with the quadratic form given by $Q((w, \varphi))=$ $\varphi(w)$ for any $w \in W$ and $\varphi \in \hat{W}$. Taking the given basis in $W$ and its "dual" in $\hat{W}$ it is seen that $V$ is an orthogonal sum of hyperbolic planes with a number of summands equal again to the cardinality of $I$. Therefore $(U, q)$ and $(V, Q)$ are isomorphic. Consider now the bilinear map

$$
\begin{aligned}
& \lambda: U \times V \longrightarrow V, \\
& (a,(w, \varphi)) \mapsto\left(\mu_{a}(w), \mu_{a}^{*}(\varphi)\right) .
\end{aligned}
$$

Then

$$
\begin{aligned}
Q\left(\mu_{a}(w), \mu_{a}^{*}(\varphi)\right) & =\mu_{a}^{*}(\varphi)\left(\mu_{a}(w)\right)=\varphi\left(\mu_{\bar{a}}\left(\mu_{a}(w)\right)\right) \\
& =\varphi(\bar{a}(a w))=q(a) \varphi(w) \\
& =q(a) Q((w, \varphi))
\end{aligned}
$$

for any $a \in U$ and $(w, \varphi) \in V$, where we have used that $\bar{a} a=q(a, e) a-a^{2}=q(a) 1$ in $C(U, q, e)$. Given an isomorphism $\psi:(U, q) \rightarrow(V, Q)$, the multiplication in $U$ defined by

$$
a b=\psi^{-1}(\lambda(a, \psi(b)))
$$

verifies $q(a b)=q(a) q(b)$ for any $a, b \in U$ and $e b=\psi^{-1}(\lambda(e, \psi(b)))=\psi^{-1} \psi(b)=b$ for any $b \in U$, so the left multiplication by $e$ is the identity and, in particular, invertible.

This example immediately implies the following:

Corollary. Over any ground field there are composition algebras with left unit with strictly nondegenerate quadratic form of arbitrary infinite dimension.

Over fields of characteristic $\neq 2$, taking into account the isomorphism between $C(U, q, e)$ and $C\left(U^{\prime},-q\right)$ mentioned in the paragraph previous to Theorem 4, easier examples may be constructed:

Example 2. Assume the characteristic of $F$ is $\neq 2$ and let $U=F e \oplus\left(\oplus_{i \in I} F e_{i}\right)$ with an infinite well ordered set $I$, and let $q$ be the quadratic form given by $q(e)=1=$ $q\left(e_{i}\right)$ for any $i \in I$ and $q\left(e, e_{i}\right)=0=q\left(e_{i}, e_{j}\right)$ for any $i \neq j$. Then $U^{\prime}=\oplus_{i \in I} F e_{i}$. Consider the (usual) Clifford algebra $C\left(U^{\prime},-q\right)=V$ which has a basis formed by the elements

$$
e_{\alpha}=e_{i_{1}} \cdots e_{i_{r}}
$$

where $r \geq 0, i_{1}<\cdots<i_{r}, \alpha=\left(i_{1}, \ldots, i_{r}\right)$ and the empty product $(r=0)$ must be understood as 1. Again the dimension of $V$ is the cardinality of $I$. We define the quadratic form $Q$ on $V$ by imposing that the elements of this basis are orthogonal and $q(a)=1$ for any $a$ in the basis. Then $(U, q) \cong(V, Q)$. Finally define $\lambda: U \rightarrow \operatorname{End}_{F}(V), a \mapsto \lambda_{a}$, by $\lambda_{e}=1$ and $\lambda_{a}$ is the left multiplication by $a$ in $C\left(U^{\prime},-q\right)$ for any $a \in U^{\prime}$, and consider the associated multiplication $\lambda: U \times V \rightarrow V$ given by $\lambda(a, v)=a \cdot v=\lambda_{a}(v)$ for any $a \in U$ and $v \in V$. From the multiplication in 
$C\left(U^{\prime},-q\right)$ it follows that $Q\left(\lambda_{e_{i}}\left(e_{\alpha}\right), e_{\beta}\right)=-Q\left(e_{\alpha}, \lambda_{e_{i}}\left(e_{\beta}\right)\right)$ for any $\alpha=\left(i_{1}, \ldots, i_{r}\right)$ and $\beta=\left(j_{1}, \ldots, j_{s}\right)$. Thus $\lambda_{e_{i}}^{*}=-\lambda_{e_{i}}=\lambda_{\bar{e}_{i}}$ and by Theorem 4 we conclude that $Q(a \cdot v)=q(a) Q(v)$ for any $a \in U$ and $v \in V$. Again, considering an isomorphism $\psi:(U, q) \rightarrow(V, Q)$ and defining the product $a b=\psi^{-1}\left(\lambda_{a}(\psi(b))\right)$ for any $a, b \in U$ we obtain a composition algebra with left unit $e$ of dimension the cardinality of $I$.

Remarks. i) Notice that if $F$ is a formally real field (see [L, page 233]), the quadratic form $q$ in Example 2 does not represent 0, so the left multiplication by any nonzero element is invertible (Theorem 3); that is, the algebra constructed is a left division algebra.

ii) With $F=\mathbb{R}$, the real field, the construction in Example 2 gives absolute valued algebras of arbitrary infinite dimension, in contrast with the examples in $[\mathrm{RP}]$ where Hilbertian dimension is used.

\section{ACKNOWLEDGMENT}

The authors are indebted to the referee for the careful revision of the paper and the many suggestions, both mathematical and about style, offered.

\section{REFERENCES}

[A-H] B.N. Allison and W. Hein, Isotopes of Some Nonassociative Algebras with Involution, J. Algebra 69 (1981), 120-142. MR 82k:17013

[A] A.A. Albert, Absolute valued algebraic algebras, Bull. Amer. Math. Soc. 55 (1949), 763768. MR 11:76h

[C] J.A. Cuenca, One-sided infinite-dimensional normed real algebras, Publ. Mat. 36 (1992), 485-488. MR 94c:47095

[E-M] A. Elduque and H.C. Myung, On flexible composition algebras, Commun. Algebra 21 (1993), 2481-2505. MR 94h:17001

[ElM-M] M.L. El-Mallah and A. Micali, Sur les dimensions des algèbres absolument valuées, J. Algebra 68 (1981), 237-246. MR 82e:17001

[F] J.M. Faillétaz, Compositions d'Espaces Bilinéaires par des Espaces Quadratiques, Doctoral dissertation, Université de Lausanne, 1992.

[H1] A. Hurwitz, Uber die Komposition der quadratischen Formen von beliebig vielen Variabeln, Nachr. Ges. Wiss. Göttingen (1898), 309-316.

[H2] A. Hurwitz, Uber die Komposition der quadratischen Formen, Math. Ann. 88 (1923), $1-25$.

[J] N. Jacobson, Structure theory of Jordan algebras, The University of Arkansas Lecture Notes 5, Fayetteville, 1981. MR 83b:17015

[Ju] J. Junker, Das Hurwitz Problem für quadratische Formen über Körper der Charakteristik 2, Diplom thesis, Saarbrücken, 1980.

[K] I. Kaplansky, Infinite-dimensional quadratic forms admitting composition, Proc. Amer. Math. Soc. 4 (1953), 956-960. MR 15:596g

[L] T.Y. Lam, The Algebraic Theory of Quadratic Forms, Benjamin, New York, 1973. MR 53:277

[E-R] J. Lawrynowicz and J. Rembielinski, On the composition of nondegenerate quadratic forms with an arbitrary index, Ann. Fac. Sci. Toulouse 10 (1989), 141-168. MR 93m: 15043

[M] H.C. Myung, Non-Unital Composition Algebras, Research Institute of Mathematics, Global Analysis Research Center, Lecture Notes Series no. 22, Seoul, 1994. MR 95f: 17003

[R] J. Radon, Lineare scharen orthogonale Matrizen, Abh. Math. Sem. Univ. Hamburg 1 (1922), 1-14.

[RP] A. Rodríguez Palacios, One-sided division absolute valued algebras, Publ. Mat. 36 (1992), 925-954. MR 94j:46048

[S1] D.B. Shapiro, Spaces of Similarities I: The Hurwitz Problem, J. Algebra 46 (1977), 148-170. MR 56:11898a 
[S2] 근 Spaces of Similarities II, Pfister Factors, J. Algebra 46 (1977), 171-181. MR 56:11898b

[S3] 근 Spaces of Similarities IV: $(s, t)$-families, Pacific J. Math. 69 (1977), $223-244$. MR 57:16193

[S4] Composition of Quadratic Forms, (manuscript).

[U-W] K. Urbanik and F.B. Wright, Absolute-valued algebras, Proc. Amer. Math. Soc. 11 (1960), 861-866. MR 22:11021

[W-S] A.R. Wadsworth and D.B. Shapiro, Spaces of Similarities III: Function Fields, J. Algebra 46 (1977), 182-188. MR 56:11898c

[W] F.B. Wright, Absolute valued algebras, Proc. Nat. Acad. Sci. USA 39 (1953), 330-332. MR 14:944b

[ZSSS] K.A. Zhevlakov, A.M. Slin'ko, I.P. Shestakov and A.I. Shirshov, Rings that are Nearly Associative, Academic Press, New York, 1982. MR 83i:17001

[Z] R. Züger, Kompositionen von quadratischen Formen mit quadratischen, bilinearen oder hermiteschen Formen, Doctoral dissertation, Universität Bern, 1995.

Departamento de Matemáticas, Universidad de Zaragoza, 50009 Zaragoza, Spain

E-mail address: jomperez@posta.unizar.es

Current address, A. Elduque: Departamento de Matemáticas y Computación, Universidad de La Rioja, 26004 Logroño, Spain

E-mail address: aelduque@dmc.unirioja.es 